\title{
2-loop additive mass renormalization with clover fermions and Symanzik improved gluons
}

\section{Apostolos Skouroupathis*}

Department of Physics, University of Cyprus, Nicosia, CY-1678, Cyprus

E-mail: php4as01@ucy.ac.cy

\section{Martha Constantinou}

Department of Physics, University of Cyprus, Nicosia, CY-1678, Cyprus

E-mail: phpgmc1@ucy.ac.cy

\section{Haralambos Panagopoulos}

Department of Physics, University of Cyprus, Nicosia, CY-1678, Cyprus

E-mail: haris@ucy.ac.cy

\begin{abstract}
We calculate the critical value of the hopping parameter, $\kappa_{c}$, in Lattice QCD, up to two loops in perturbation theory. We employ the Sheikholeslami-Wohlert (clover) improved action for Wilson fermions and the Symanzik improved gluon action for 4- and 6-link loops.

The quantity which we study is a typical case of a vacuum expectation value resulting in an additive renormalization; as such, it is characterized by a power (linear) divergence in the lattice spacing, and its calculation lies at the limits of applicability of perturbation theory.

Our results are polynomial in $c_{\mathrm{SW}}$ (clover parameter) and cover a wide range of values for the Symanzik coefficients $c_{i}$. Furthermore, the dependence on the number of colors $N$ and the number of fermionic flavors $N_{f}$ is shown explicitly. In order to compare our results to non perturbative evaluations of $\kappa_{c}$ coming from Monte Carlo simulations, we employ an improved perturbation theory method applied to improved actions.
\end{abstract}

XXIV International Symposium on Lattice Field Theory

July 23-28 2006

Tucson Arizona, US

* Speaker. 


\section{Introduction}

In the present work, we study clover fermions [1] and Symanzik improved gluons [2], for the calculation of the critical value of the hopping parameter, $\kappa_{c}$ in perturbative Lattice QCD, to two loops order in a perturbative expansion.

Concerning gauge fields, we employ the Symanzik improved action [2]. The purpose of introducing such an improvement of the lattice action, is to minimize the consequences of a non-zero lattice spacing, order by order in perturbation theory. Several choices are made for the coefficients of the action corresponding to values which are most often used in the literature.

The discretization of the theory via lattice regularization introduces some difficulties that do not exist in the continuum theory. To be able to recover the continuum limit, we must demand strict locality and absence of doublers. It is known that these requirements lead to breaking of chiral symmetry. Of course, while approaching the continuum limit we expect to recover chirality. This is the point where one must introduce the hopping parameter. The main idea is to ensure chiral symmetry, by setting the renormalized fermionic mass $\left(m_{R}\right)$ equal to zero. Because of additive renormalization, setting the bare fermionic mass equal to zero is not enough. Hence, there is a critical mass, the role of which is to guarantee that $m_{R}$ vanishes. This quantity is directly related to the hopping parameter $\kappa$. Its critical value $\left(\kappa_{c}\right)$ is responsible for restoring chiral symmetry.

Previous works on the hopping parameter and its critical value appear in the literature for Wilson fermions-plaquette action gluons [3] and for clover fermions-plaquette action gluons [4]. The procedure and notation in our work is the same as in the above references.

Our results for $\kappa_{c}$ (and thus for the critical mass) depend on the number of colors $(N)$ and fermionic flavors $\left(N_{f}\right)$. Besides that, there is an explicit dependence on the clover parameter $c_{\mathrm{SW}}$ which is kept as a free parameter. The dependence of the results on the choice of Symanzik coefficients cannot be given in closed form; instead, we present it in a list of Tables and Figures.

The rest of the paper is organized as follows: In Sec. 2 we define the actions in their discretized form, as well as the connection between the hopping parameter and fermionic mass. Furthermore, there is a description of our calculations along with the necessary Feynman diagrams. In Sec. 3 our results are presented and compared with previous Monte Carlo simulations. Finally, in Sec. 4 we apply to our 1- and 2-loop results an improvement method, proposed by us [5, 6, 7]. This method resums a certain infinite class of subdiagrams, to all orders in perturbation theory, leading to improved results. A full write-up of this work, including detailed tables of results is forthcoming [8].

\section{Formulation of the Problem}

We begin with the Wilson formulation of the QCD action on the lattice, with the addition of the clover (SW) [1] term for fermions. In standard notation, it reads:

$$
\begin{aligned}
S_{L} & =S_{G}+\sum_{f} \sum_{x}(4 r+m) \bar{\psi}_{f}(x) \psi_{f}(x) \\
& -\frac{1}{2} \sum_{f} \sum_{x, \mu}\left[\bar{\psi}_{f}(x)\left(r-\gamma_{\mu}\right) U_{\mu}(x) \psi_{f}(x+\mu)+\bar{\psi}_{f}(x+\mu)\left(r+\gamma_{\mu}\right) U_{\mu}(x)^{\dagger} \psi_{f}(x)\right] \\
& +\frac{i}{4} c_{\mathrm{SW}} \sum_{f} \sum_{x, \mu, v} \bar{\psi}_{f}(x) \sigma_{\mu v} \hat{F}_{\mu v}(x) \psi_{f}(x),
\end{aligned}
$$




$$
\begin{aligned}
& \text { where: } \hat{F}_{\mu v} \equiv \frac{1}{8 a^{2}}\left(Q_{\mu v}-Q_{v \mu}\right) \\
& \text { and: } Q_{\mu \nu}=U_{x, x+\mu} U_{x+\mu, x+\mu+\nu} U_{x+\mu+v, x+\nu} U_{x+v, x}+U_{x, x+\nu} U_{x+v, x+v-\mu} U_{x+\nu-\mu, x-\mu} U_{x-\mu, x} \\
& +U_{x, x-\mu} U_{x-\mu, x-\mu-\nu} U_{x-\mu-v, x-\nu} U_{x-v, x}+U_{x, x-\nu} U_{x-v, x-v+\mu} U_{x-v+\mu, x+\mu} U_{x+\mu, x}
\end{aligned}
$$

The clover coefficient $c_{\mathrm{SW}}$ is treated here as a free parameter; $r$ is the Wilson parameter; $f$ is a flavor index; $\sigma_{\mu \nu}=(i / 2)\left[\gamma_{\mu}, \gamma_{v}\right]$. Powers of the lattice spacing $a$ have been omitted.

Regarding gluons, we use the Symanzik action, involving Wilson loops with 4 and 6 links:

$$
\begin{aligned}
S_{G}=\frac{2}{g^{2}} & {\left[c_{0} \sum_{\text {plaq }} \operatorname{Re} \operatorname{Tr}\left(1-U_{\text {plaq }}\right)+c_{1} \sum_{\text {rect }} \operatorname{Re} \operatorname{Tr}\left(1-U_{\text {rect }}\right)\right.} \\
& \left.+c_{2} \sum_{\text {chair }} \operatorname{Re} \operatorname{Tr}\left(1-U_{\text {chair }}\right)+c_{3} \sum_{\text {paral }} \operatorname{Re} \operatorname{Tr}\left(1-U_{\text {paral }}\right)\right]
\end{aligned}
$$

The correct classical continuum limit requires: $c_{0}+8 c_{1}+16 c_{2}+8 c_{3}=1$. The full action is: $S=S_{L}+S_{g f}+S_{g h}+S_{m}$., where $S_{g f}, S_{g h}, S_{m}$ are standard gauge fixing, ghost and measure terms.

The bare fermionic mass $m_{B}$ must be set to zero for chiral invariance in the classical continuum limit. The value of the parameter $c_{\mathrm{SW}}$ and of the Symanzik coefficients $c_{i}$ can be chosen arbitrarily; they are normally tuned in a way as to minimize $\mathscr{O}(a)$ effects. Terms proportional to $r$ in the action, as well as the clover terms, break chiral invariance. They vanish in the classical continuum limit; at the quantum level, they induce nonvanishing, flavor-independent corrections to the fermion masses. Numerical simulation algorithms usually employ the hopping parameter,

$$
\kappa \equiv \frac{1}{2 m_{B} a+8 r}
$$

as an adjustable quantity. Its critical value, at which chiral symmetry is restored, is thus $1 / 8 r$ classically, but gets shifted by quantum effects.

We denote by $d m$ the perturbative contribution that must be added to the bare mass, in order to lead to zero renormalized mass. At tree level, $m_{B}=0$.

$$
d m=d m_{(1-\mathrm{loop})}+d m_{(2-\mathrm{loop})}
$$

Two diagrams contribute to $d m_{(1-\text { loop })}$, shown in Figure 1 . The quantity $d m_{(2-\text { loop })}$ receives contributions from a total of 26 diagrams, shown in Figure 2. Genuine 2-loop diagrams must be evaluated at $m_{B} \rightarrow 0$; in addition, one must include to this order the 1-loop diagram containing an $\mathscr{O}\left(g^{2}\right)$ mass counterterm (diagram 23). Certain sets of diagrams, corresponding to renormalization of 1-loop propagators, must be evaluated together in order to obtain an infrared-convergent result: These are diagrams $7+8+9+10+11,12+13,14+15+16+17+18,19+20,21+22+23$.

\section{Numerical Results}

We have selected a set of most widely used values for the Symanzik coefficients, shown in Table 1. (In all these cases, $c_{2}=0$. In general, for given values of $C_{1} \equiv c_{2}+c_{3}, C_{2} \equiv c_{1}-c_{2}-c_{3}$ the dependence on $c_{2}$ is polynomial and thus we need not choose a numerical value for it.) 


\begin{tabular}{llll}
\hline \hline \multicolumn{1}{c}{ Action } & \multicolumn{1}{c}{$c_{0}$} & \multicolumn{1}{c}{$c_{1}$} & \multicolumn{1}{c}{$c_{3}$} \\
\hline \hline Plaquette & 1.0 & 0.0 & 0.0 \\
Symanzik & 1.6666667 & -0.083333 & 0.0 \\
TILW, $\beta c_{0}=8.60$ & 2.3168064 & -0.151791 & -0.0128098 \\
TILW, $\beta c_{0}=8.45$ & 2.3460240 & -0.154846 & -0.0134070 \\
TILW, $\beta c_{0}=8.30$ & 2.3869776 & -0.159128 & -0.0142442 \\
TILW, $\beta c_{0}=8.20$ & 2.4127840 & -0.161827 & -0.0147710 \\
TILW, $\beta c_{0}=8.10$ & 2.4465400 & -0.165353 & -0.0154645 \\
TILW, $\beta c_{0}=8.00$ & 2.4891712 & -0.169805 & -0.0163414 \\
Iwasaki & 3.648 & -0.331 & 0.0 \\
DBW2 & 12.2688 & -1.4086 & 0.0 \\
\hline \hline
\end{tabular}

Table 1: Input parameters $c_{0}, c_{1}, c_{3}$.
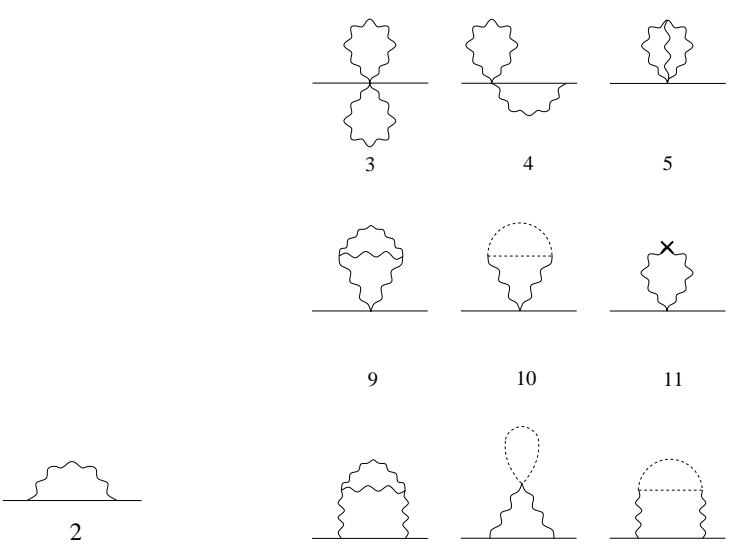

Figure 1: One-loop diagrams contributing to $d m_{(1-\text { loop })}$. Wavy (solid) lines represent gluons (fermions).

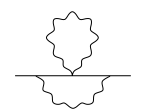

21

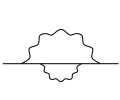

22

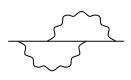

27
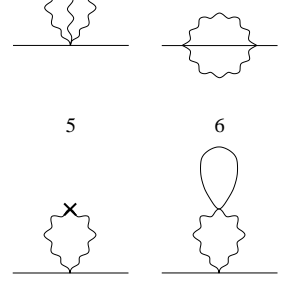

10

11
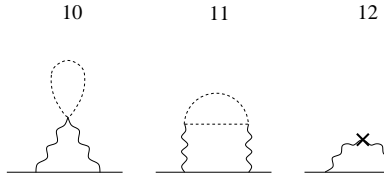

16

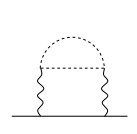

17

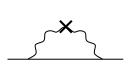

18

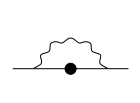

23

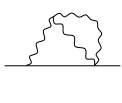

24

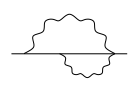

25

Figure 2: Two-loop diagrams contributing to $d m_{(2-\text { loop })}$. Wavy (solid, dotted) lines represent gluons (fermions, ghosts). Crosses denote vertices stemming from the measure part of the action; a solid circle is a fermion mass counterterm. 
The contribution of the $l^{\text {th }}$ 1-loop diagram to $d m$, can be expressed by:

$$
d_{l}=\frac{\left(N^{2}-1\right)}{N} g^{2} \cdot \sum_{i=0}^{2} c_{\mathrm{SW}}^{i} \varepsilon_{l}^{(i)}
$$

where $\varepsilon_{l}^{(i)}$ are numerical constants and their values depend on the Symanzik coefficients $c_{i}$. The dependence on $c_{\mathrm{SW}}$ is seen to be polynomial of degree $2(i=0,1,2)$.

The contribution of 2-loop diagrams without closed fermion loops takes the form

$$
d_{l}=\frac{\left(N^{2}-1\right)}{N^{2}} g^{4} \cdot \sum_{i, j, k} c_{\mathrm{SW}}^{i} N^{j} c_{2}^{k} e_{l}^{(i, j, k)}
$$

where the index $l$ runs over all contributing diagrams, $j=0,2$ and $k=0,1,2$. The coefficients $e_{l}^{(i, j, k)}$ exhibit a further dependence on Symanzik coefficients (only through the combinations $C_{1}, C_{2}$ ), which cannot be expressed in closed form and is presented numerically in what follows.

The contribution of 2-loop diagrams, containing closed fermion loops, has the form

$$
d_{l}=\frac{\left(N^{2}-1\right)}{N} N_{f} g^{4} \cdot \sum_{i=0}^{4} c_{S W}^{i} \tilde{e}_{l}^{(i)}
$$

In order to enable cross-checks, numerical per-diagram values of the constants $\varepsilon_{l}^{(i)}, e_{l}^{(i, j, k)}$ and $\tilde{e}_{l}^{(i)}$ are presented in our forthcoming publication [8], for the Iwasaki action.

The total contribution of 1-loop diagrams, for $N=3$, can be written as a polynomial function of the clover parameter $c_{\mathrm{SW}}$. For the Wilson and Iwasaki actions we find, respectively:

$$
\begin{aligned}
& d m_{(1-\text { loop })}^{\text {Wilson }}=g^{2}\left(-0.434285489(1)+0.1159547570(3) c_{\mathrm{SW}}+0.0482553833(1) c_{\mathrm{SW}}^{2}\right) \\
& d m_{(1-\text { loop })}^{\text {Iwasaki }}=g^{2}\left(-0.2201449497(1)+0.0761203698(3) c_{\mathrm{SW}}+0.0262264231(1) c_{\mathrm{SW}}^{2}\right)
\end{aligned}
$$

To illustrate our 2-loop results for some particular choices of the action, we set $N=3, c_{2}=0$ and we use three different values for the flavour number: $N_{f}=0,2$. Thus, for the Wilson action:

$$
\begin{array}{r}
N_{f}=0: \quad d m_{(2-\text { loop })}=g^{4}\left(-0.1255626(2)+0.0203001(2) c_{\mathrm{SW}}+0.00108420(7) c_{\mathrm{SW}}^{2}\right. \\
\left.-0.00116538(2) c_{\mathrm{SW}}^{3}-0.0000996725(1) c_{\mathrm{SW}}^{4}\right) \\
N_{f}=2: \quad d m_{(2-\text { loop })}=g^{4}\left(-0.1192361(2)+0.0173870(2) c_{\mathrm{SW}}+0.00836498(8) c_{\mathrm{SW}}^{2}\right. \\
\left.-0.00485727(3) c_{\mathrm{SW}}^{3}-0.0011561947(4) c_{\mathrm{SW}}^{4}\right)
\end{array}
$$

and for the Iwasaki action:

$$
\begin{gathered}
N_{f}=0: \quad d m_{(2-\text { loop })}=g^{4}\left(-0.0099523(2)-0.0024304(5) c_{\mathrm{SW}}-0.00232855(4) c_{\mathrm{SW}}^{2}\right. \\
\left.-0.00032100(2) c_{\mathrm{SW}}^{3}-0.0000419365(1) c_{\mathrm{SW}}^{4}\right) \\
N_{f}=2: \quad d m_{(2-\text { loop })}=g^{4}\left(-0.0076299(2)-0.0040731(5) c_{\mathrm{SW}}+0.00102758(6) c_{\mathrm{SW}}^{2}\right. \\
\left.-0.00242924(3) c_{\mathrm{SW}}^{3}-0.000457690(2) c_{\mathrm{SW}}^{4}\right)
\end{gathered}
$$

In Figures 3 and 4 we present the values of $d m_{(2-\text { loop })}$ for $N_{f}=0,2$, respectively; the results are shown for all our choices of Symanzik actions, as a function of $c_{\mathrm{SW}}\left(N=3, c_{2}=0\right)$. 


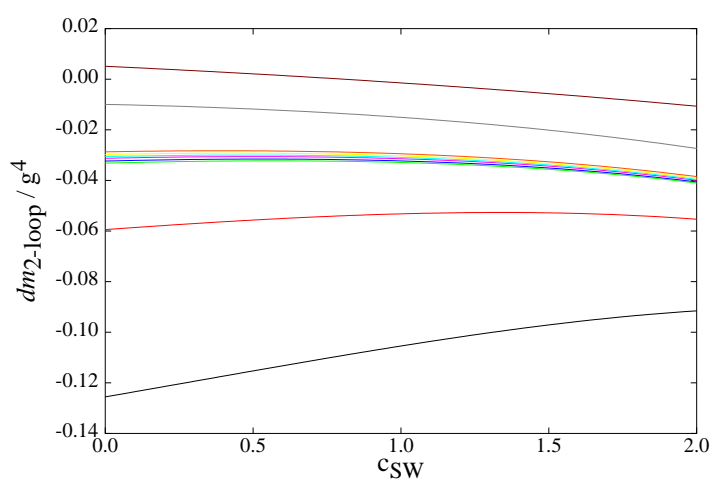

Figure 3: Total contribution of 2-loop diagrams, for $N=3, N_{f}=0$ and $c_{2}=0$. Actions (top to bottom): DBW2, Iwasaki, $\operatorname{TILW}(\beta=8.00,8.10$, 8.20, 8.30, 8.45, 8.60), Symanzik, Plaquette.

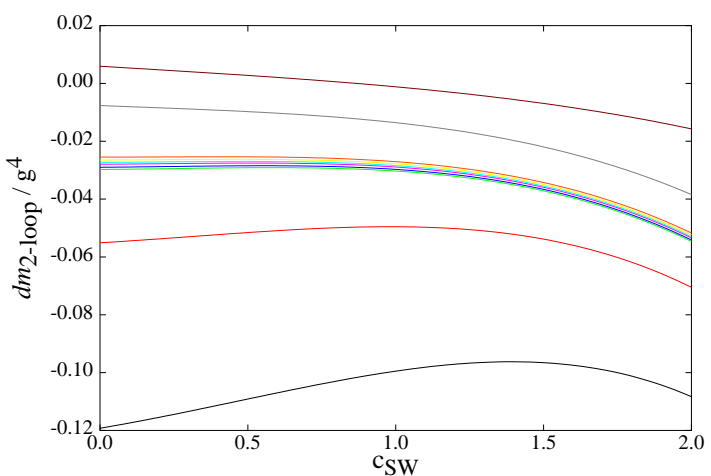

Figure 4: Total contribution of 2-loop diagrams, for $N=3, N_{f}=2$ and $c_{2}=0$. Actions (top to bottom): DBW2, Iwasaki, $\operatorname{TILW}(\beta=8.00$, 8.20, 8.30, 8.45, 8.60), Symanzik, Plaquette.

\section{Improved Perturbation Theory}

We now apply our method of improving perturbation theory [7], based on resummation of an infinite subset of tadpole diagrams, termed "cactus" diagrams. In Ref. [7] we show how this procedure provides a simple way of dressing (to all orders) perturbative results at any given order (such as the 1- and 2-loop results of the present calculation). Some alternative ways of improving perturbation theory have been proposed in Refs. [9, 10]. In a nutshell, our procedure involves replacing the original values of the Symanzik and clover coefficients by improved values, which are explicitly computed in [7]. Taking also due care to avoid double counting of diagrams, we calculate the improved ("dressed") value $d m^{\mathrm{dr}}$ of the critical mass $\left(N=3, c_{2}=0\right)$.

We choose to study the case of the Wilson action $(\beta=5.29)$ and the Iwasaki action $(\beta=1.95)$ with $N=3$ and $N_{f}=2$. Using these values, the contribution to $d m_{(2-\text { loop })}^{\mathrm{dr}}$ is a polynomial of $c_{\mathrm{SW}}$ :

$$
\begin{aligned}
d m_{(2-\text { loop }), \text { Wilson }}^{\mathrm{dr}}= & -0.77398(5)+0.1632999(3) c_{\mathrm{SW}}+0.06225(2) c_{\mathrm{SW}}^{2} \\
& -0.00440064(8) c_{\mathrm{SW}}^{3}-0.000737797(1) c_{\mathrm{SW}}^{4} \\
d m_{(2-\text { loop}), \text { Iwasaki }}^{\mathrm{dr}}= & -0.0813302(9)+0.043030(3) c_{\mathrm{SW}}+0.0308196(2) c_{\mathrm{SW}}^{2} \\
& -0.00767090(8) c_{\mathrm{SW}}^{3}-0.001160923(1) c_{\mathrm{SW}}^{4}
\end{aligned}
$$

The comparison between the total dressed contribution $d m^{\mathrm{dr}}=d m_{(1-\text { loop })}^{\mathrm{dr}}+d m_{(2-\text { loop })}^{\mathrm{dr}}$ and the unimproved contribution, $d m$, for the plaquette action $\left(\beta=5.29, N_{f}=2\right)$ is exhibited in Figure 5, as a function of $c_{\mathrm{SW}}$. Similarly, $d m$ for the Iwasaki action $\left(\beta=1.95, N_{f}=2\right)$ is shown in Figure 6.

\begin{tabular}{lcclccccc}
\hline \hline \multicolumn{1}{c}{ Action } & $N_{f}$ & $\beta$ & \multicolumn{1}{c}{$c_{\mathrm{SW}}$} & $\kappa_{1-\text { loop }}$ & $\kappa_{2-\text { loop }}$ & $\kappa_{1-\text { loop }}^{\mathrm{dr}}$ & $\kappa_{2-\text { loop }}^{\mathrm{dr}}$ & $\kappa_{\text {cr }}^{\text {non-pert }}$ \\
\hline \hline Plaquette & 0 & 6.00 & 1.479 & 0.1301 & 0.1335 & 0.1362 & 0.1362 & 0.1392 \\
Plaquette & 0 & 6.00 & 1.769 & 0.1275 & 0.1306 & 0.1337 & 0.1332 & 0.1353 \\
Plaquette & 2 & 5.29 & 1.9192 & 0.1262 & 0.1307 & 0.1353 & 0.1341 & 0.1373 \\
Iwasaki & 2 & 1.95 & 1.53 & 0.1292 & 0.1368 & 0.1388 & 0.1379 & 0.1421 \\
\hline \hline
\end{tabular}

Table 2: 1- and 2-loop results, and non-perturbative estimates for $\kappa_{\mathrm{cr}}$ 
Finally, in Table 2, we present a comparison of dressed and undressed results with non perturbative estimates for $\kappa_{\mathrm{cr}}[11,12,13]$. We observe that improved perturbation theory, applied to 1-loop results, already leads to a much better agreement with the non perturbative estimates.

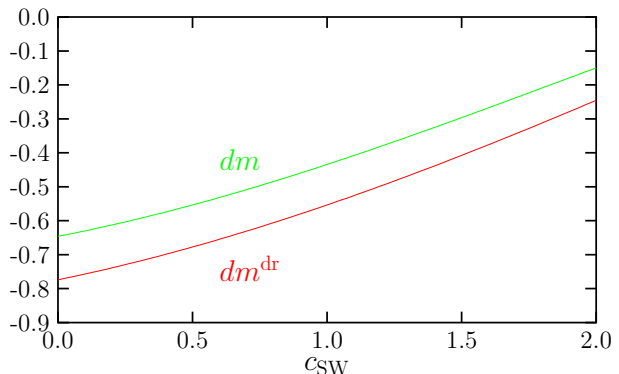

Figure 5: Improved and unimproved values of $d m$ as a function of $c_{\mathrm{SW}}$, for the plaquette action.

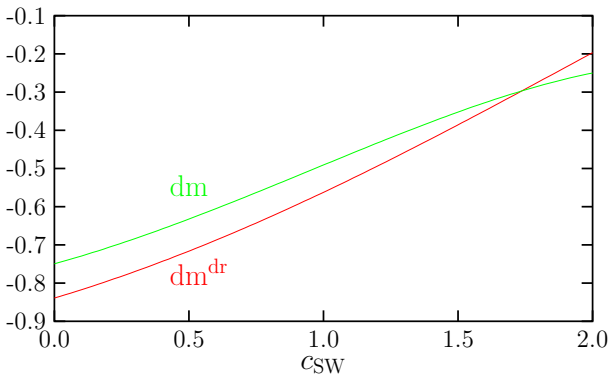

Figure 6: Improved and unimproved values of $d m$ as a function of $c_{\mathrm{SW}}$, for the Iwasaki action.

Acknowledgements: Work supported in part by the Research Promotion Foundation of Cyprus.

\section{References}

[1] B. Sheikholeslami and R. Wohlert, Improved continuum limit lattice action for QCD with wilson fermions, Nucl. Phys. B259 (1985) 572.

[2] K. Symanzik, Continuum limit and improved action in lattice theories, Nucl. Phys. B226 (1983) 187; Nucl. Phys. B226 (1983) 205.

[3] E. Follana and H. Panagopoulos, The Critical Mass of Wilson Fermions: A Comparison of Perturbative and Monte Carlo Results, Phys. Rev. D63 (2001) 017501.

[4] H. Panagopoulos and Y. Proestos, The Critical Hopping Parameter in $\mathscr{O}($ a) improved Lattice QCD, Phys. Rev. D65 (2001) 014511.

[5] H. Panagopoulos and E. Vicari, Resummation of Cactus Diagrams in Lattice QCD, Phys. Rev. D58 (1998) 114501.

[6] H. Panagopoulos and E. Vicari, Resummation of Cactus Diagrams in the Clover Improved Lattice Formulation of QCD, Phys. Rev. D59 (1999) 057503.

[7] M. Constantinou, H. Panagopoulos and A. Skouroupathis, Improved Perturbation Theory for Improved Lattice Actions, Phys. Rev. D74 (2006) 074503 [hep-lat/0606001].

[8] A. Skouroupathis, M. Constantinou and H. Panagopoulos, In preparation.

[9] G. Parisi, in: High-Energy Physics - 1980, XX Int. Conf., Madison (1980), ed. L. Durand and L. G. Pondrom (American Institute of Physics, New York, 1981).

[10] G. P. Lepage and P. B. Mackenzie, On the Viability of Lattice Perturbation Theory, Phys. Rev. D48 (1993) 2250.

[11] M. Lüscher, S. Sint, R. Sommer, P. Weisz and U. Wolff, Non-perturbative O(a) improvement of lattice QCD, Nucl. Phys. B491 (1997) 323.

[12] K. Jansen and R. Sommer, $\mathscr{O}(a)$ improvement of lattice $Q C D$ with two flavors of Wilson quarks, Nucl. Phys. B530 (1998) 185.

[13] UKQCD Collaboration (C.R. Allton et al.), Effects of non-perturbatively improved dynamical fermions in QCD at fixed lattice spacing, Phys. Rev. D65 (2002) 054502 [hep-lat/0107021]. 